\title{
Giant Coronary Artery Pseudoaneurysm after Drug-Eluting Stent Implantation
}

\author{
Ashok Bhupali', Amit Joshi', Sachin Patil2, Prithiviraj Jadhav², Sayi Prasad, \\ Tanmay Vora ${ }^{1}$, Nirlep Gajiwala ${ }^{3}$, Ashok Thakkar ${ }^{3}$ \\ ${ }^{1}$ Apple Hospitals \& Research Institute Ltd., Kolhapur, India \\ ${ }^{2}$ Aster Aadhar Hospital, Kolhapur, India \\ ${ }^{3}$ Sahajanand Medical Technologies Pvt. Ltd., Surat, India \\ Email: abhupali@gmail.com
}

Received 29 June 2015; accepted 10 August 2015; published 13 August 2015

Copyright (C) 2015 by authors and Scientific Research Publishing Inc.

This work is licensed under the Creative Commons Attribution International License (CC BY).

http://creativecommons.org/licenses/by/4.0/

c) (7) Open Access

\begin{abstract}
Formation of coronary artery pseudoaneurysm after percutaneous coronary intervention is rarely reported. A close review of available literature indicates a trend of growing incidence in recent times. Here, we present a case of coronary pseudoaneurysm developing in the left circumflex artery within 50 days of sirolimus-eluting stent implantation in a 47-year-old man who was treated for triple-vessel coronary artery disease. Initially, the patient refused any further treatment. However, considering the rapid growth of pseudoaneurysm and increased severity of symptoms at subsequent follow-up, we decided to treat giant pseudoaneurysm with embolization coils. The procedure was successful and the patient remained asymptomatic thereafter.
\end{abstract}

\section{Keywords}

Coronary Aneurysm, Drug-Eluting Stent, Embolization Coil, Interventional Cardiology, Pseudoaneurysm, Sirolimus

\section{Introduction}

Development of coronary artery aneurysm after percutaneous coronary intervention is a rare entity, with reported incidence of about $0.3 \%$ to $6.0 \%$. Majority of these aneurysms are suspected to be pseudoaneurysm rather than true aneurysm [1]. The causative factors include residual dissection and deep arterial-wall injury secondary to the use of oversized balloons/stents, high-pressure balloon inflation, atherectomy, and laser angioplasty [2]. A close review of available literature suggests a growing incidence of coronary artery aneurysms after implantation of drug-eluting stent (DES) in recent times. The possible pathophysiological mechanisms are: a) delayed 
re-endothelialization and healing secondary to the antiproliferative action of eluted drug; b) cell necrosis or apoptosis due to antimetabolite effects of the drug; c) inflammation compounded by vascular injury (residual dissections, contained perforations) secondary to the hypersensitivity reaction to metal, polymer, or drug; or d) acquired late stent malapposition [1]-[3].

The clinical course of coronary aneurysms depends on their anatomical details (i.e. size, site of origin, and layer of the arterial wall). The onset of coronary aneurysm formation has been reported from 3 days to 4 years after the DES implantation, with a varied presentation from asymptomatic state to severe angina. Some aneurysms resolve naturally, while others may lead to life-threatening complications such as rupture, thrombosis, embolization, myocardial infarction, and even sudden death [3]. A study has reported that the incidence of coronary aneurysm after DES implantation is more common in left anterior descending artery (LAD), followed by right coronary artery (RCA), and rarely in left circumflex artery (LCX) [4]. Herein, we are reporting a case of coronary pseudoaneurysm developing in LCX vessel within 50 days of DES implantation in a 47-year-old man, who was successfully managed with embolization coils.

\section{Case Presentation}

A 47-year-old normotensive and nondiabetic male presented to our hospital with symptoms of class II angina. Echocardiography of the patient was normal, but 2-dimensional (2D) echocardiography showed concentric hypertrophy of left ventricle with no regional wall motion abnormality. The ejection fraction was $60 \%$. Patient was evaluated for angina on exertion, and the treadmill test was positive for inducible ischemia. The patient underwent coronary angiography, which revealed a triple-vessel disease. Subsequently, percutaneous coronary intervention was performed. The patient received $2.5 \times 13 \mathrm{~mm}$ sirolimus-eluting polymer-based stent in LAD, 2.5 $\times 23 \mathrm{~mm}$ sirolimus-eluting polymer-based stent in LCX, and $2.75 \times 13 \mathrm{~mm}$ plus $2.5 \times 18 \mathrm{~mm}$ sirolimus-eluting polymer-based stents in RCA (Figure 1). Patient was discharged $48 \mathrm{~h}$ after the successful stent implantations. He was on routine medications including dual antiplatelets, aspirin $300 \mathrm{mg}$ and clopidogrel $75 \mathrm{mg}$ daily.

The patient remained asymptomatic at subsequent follow-ups at our outpatient clinic. Nevertheless, he started to complain of angina after one and half month of DES implantation. Repeat coronary angiography displayed normal flow in LAD and RCA with patent stents, but LCX had total in-stent restenosis with an aneurysm-like formation at the proximal end of the stented vessel area (size: $6 \times 4 \mathrm{~mm}$; Figure 2). Additionally, a $99 \%$ lesion with thrombus burden was observed in second obtuse marginal (OM2) vessel. Patient was offered treatment either in the form of covered stent or surgical excision; however, he refused any further treatment. Subsequently, he was counseled for his condition and was discharged with optimized medical regimen comprising a statin and warfarin in addition to the dual antiplatelet therapy.

Again after 1 month, the patient reported a significant increase in the frequency and the severity of angina. He was again subjected for coronary angiography, which revealed a significant increase in the size of pseudoaneurysm at the proximal end of LCX stent (size: $15 \times 11 \mathrm{~mm}$ ). The stent was occluded and the pseudoaneurysm had extended proximally in the mid LCX compared to the earlier angiography (Figure 3). Patient's 2D echo also confirmed intramural location of pseudoaneurysm (Figure 4).

In view of increasing size and extent of the giant pseudoaneurysm, it was decided to do coiling for this lesion. Left coronary ostium was engaged by using a Launcher 7F (EBU 3) coronary guide catheter (Medtronic, USA). Then, a ProTrack microcatheter (Baylis Medical, Canada) was placed in to proximal LCX. Subsequently, two Hilal embolization coils (Cook Medicals, USA) of $3 \mathrm{~cm} \times 3 \mathrm{~mm}$ size were deployed in the proximal LCX (Figure 5). The procedure was successful. The postoperative period was uneventful, and the patient was discharged from the hospital with no change in his drug regimen. At three-month follow-up, 2D echocardiography was performed which showed no signs of coronary artery aneurysm (Figure 6). During this interval, the patient remained asymptomatic.

\section{Discussion}

Although DES have made a significant impact on interventional cardiology practice by offering extraordinarily low rates of restenosis, important safety concerns regarding their usage has been acknowledged widely. While major emphasis has been placed on the incidence of late stent thrombosis, formation of coronary artery aneurysm is emerging as another major adverse event after DES implantation [5].

Coronary aneurysms can be classified in to true aneurysm and pseudoaneurysm. A true aneurysm is characte- 

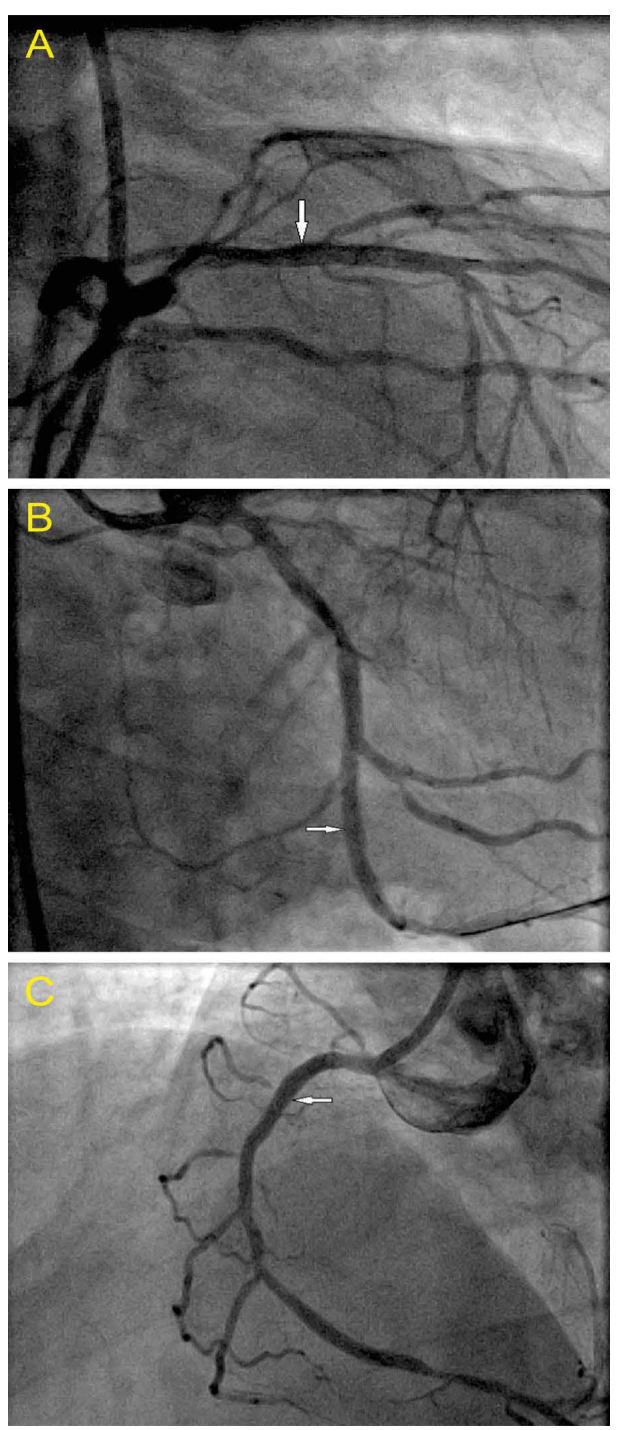

Figure 1. Implantation of (A) $2.5 \times 13 \mathrm{~mm}$ sirolimus-eluting stent to LAD, (B) $2.5 \times 23 \mathrm{~mm}$ sirolimus-eluting stent to LCX, and (C) 2.75 $\times 13 \mathrm{~mm}$ plus $2.50 \times 18 \mathrm{~mm}$ sirolimus-eluting stent to RCA.

rized as a segment with a maximal lumen area larger than a proximal reference lumen by at least $50 \%$. On the other hand, a pseudoaneurysm results from the loss of vessel wall integrity and transition from a three-layered wall to an outwardly bulging monolayer [6]. True coronary aneurysm usually occurs due to late acquired stent malapposition, while pseudoaneurysm occurs as a result of traumatic dissection or perforation of a coronary artery or due to stent fracture [6]. Based on these characteristics, the aneurysm in our case was identified as pseudoaneurysm.

Despite advances in interventional cardiology, the true incidence, clinical course, and treatment of coronary artery aneurysms after DES implantation remain largely unknown [3]. The diagnosis of coronary artery pseudoaneurysm in a patient with suspected myocardial ischemia is made on the basis of cardiac imaging procedures. Coronary angiography remains the gold standard as it not only provides information regarding the size, shape, location, and number of aneurysms, but also illustrates the presence or absence of associated stenosis. Other noninvasive tools such as transesophageal echocardiography, contrast-enhanced computed tomography and magnetic resonance imaging are also valuable for initial evaluation [3]. Since our patient presented with angina after DES implantation, he was taken up directly for diagnostic coronary angiogram. Subsequent angiographic findings and $2 \mathrm{D}$ echo images led us to suspect a pseudoaneurysm in our patient. We suggest that all patients 

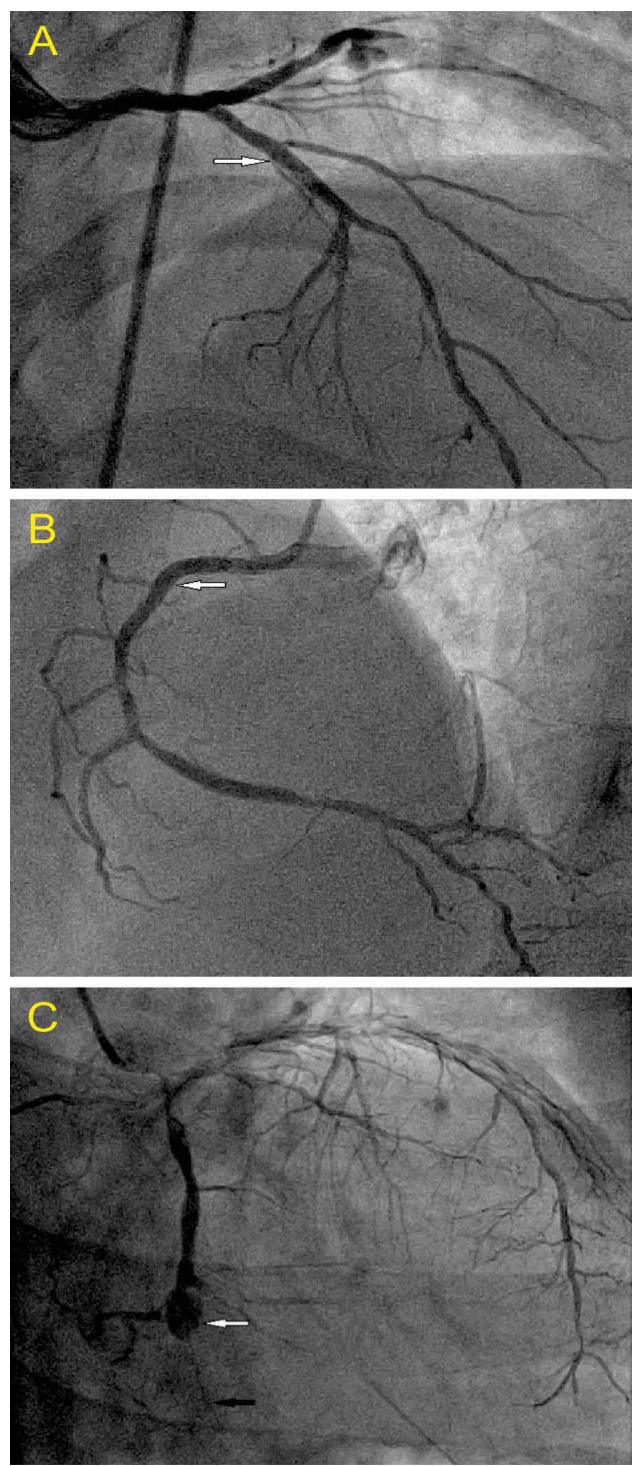

Figure 2. Repeat angiography at 45 days post-procedure showed patent stents in (A) LAD and (B) RCA, and (C) total occlusion of LCX stent with aneurysm formation at the proximal end.

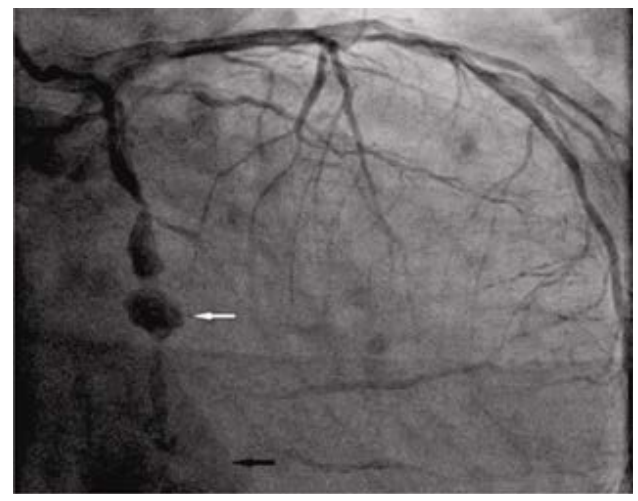

Figure 3. Subsequent angiography showing aneurysm extending in the mid LCX compared to earlier angiography. 


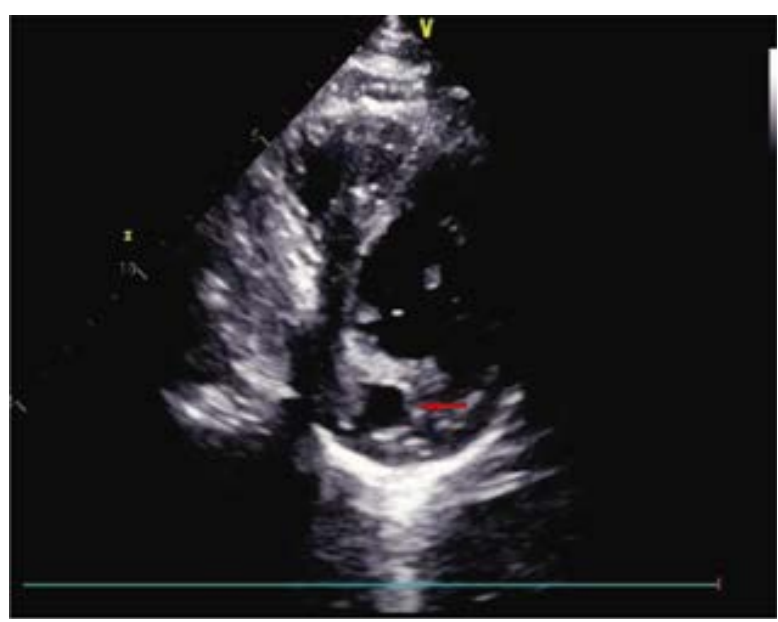

Figure 4. 2D-echocardiography indicating intramural location of pseudoaneurysm.

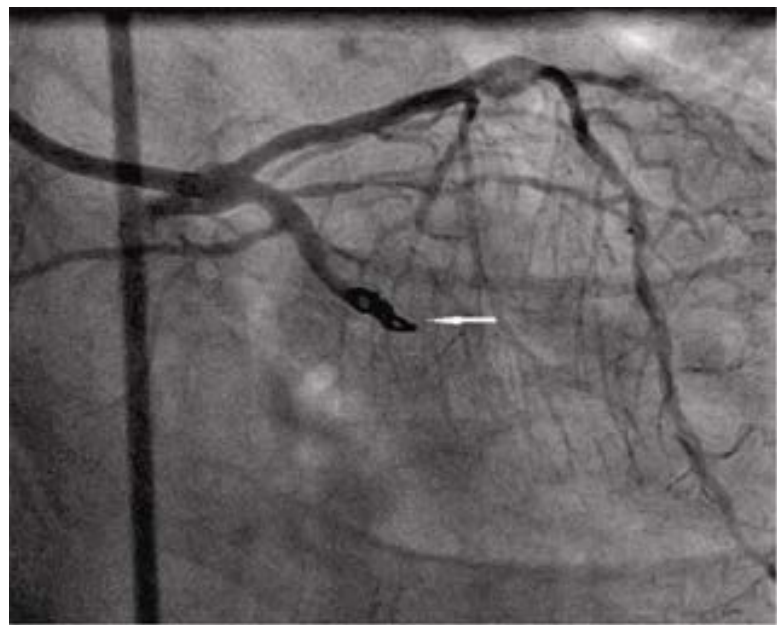

Figure 5. Deployment of two embolization coils into proximal left circumflex artery.

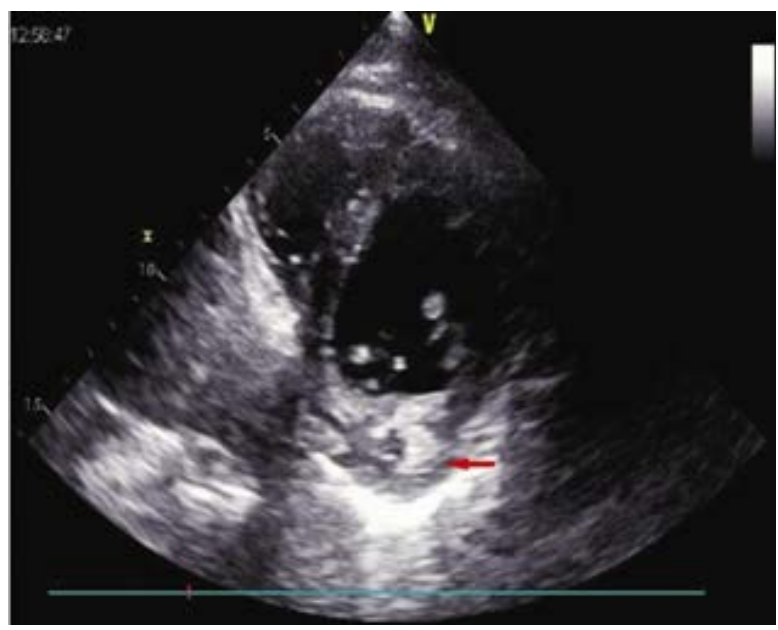

Figure 6. Follow-up 2D-echocardiography after coil embolization; patient remained asymptomatic. 
with chest pain post DES-implantation should be thoroughly evaluated for possible aneurysm formation.

Because of the rarity of this clinical phenomenon, there is no standard therapy recommended for pseudoaneurysm [6]. Several therapeutic approaches have been proposed such as conservative therapy (medical management and observation), percutaneous exclusion of the aneurismal lumen, and surgical intervention (placement of another stent, stent grafts, coronary coils, or coronary artery bypass grafting). Overall, the management of these patients is challenging and needs to be individualized based on the size and location of the aneurysm and based on the presence of endovascular thrombosis [1]. In our case, all the therapeutic options were explored. Medical therapy with clinical observation was denied because of the significant expansion of the pseudoaneurysm over time. Similarly, implantation of another bare-metal stent, vein-graft coated and covered stents might be successful for complete sealing of coronary aneurysms, but are associated with impaired navigability and high risk of restenosis at the stent edges [7]. Based on relative merits, we decided to use an embolization coils in our patient. In literature, stent-assisted coil embolization of aneurysms has been successfully performed in wide-range of intracranial aneurysms and coronary aneurysms [8] [9]. Further, a case report has demonstrated a successful treatment with endovascular coil embolization in a patient with Behçet disease-related giant aneurysm in the RCA [10]. We believe that coil embolization can be a valuable method to treat selected patients with coronary aneurysms who have a suitable anatomy, as demonstrated in our patient.

The patient in our case had received sirolimus-eluting polymer-based stents. This adds to the existing theory that sirolimus-eluting polymer-based stent may play a role in the development of pseudoaneurysm after DES placement [1]. However, sirolimus-eluting stents implanted in the LAD and RCA remained patent in our case, which supports the hypothesis that other factors might be at play. Hence, the exact mechanisms responsible for coronary pseudoaneurysm formation in this patient remain unknown. Given the rapid onset of aneurysm formation, there is a possibility that arterial injury during stent implantation might be the likely contributor to pseudoaneurysm formation rather than the chronic arterial response to the stent, polymer, and drug. Further, the coronary pseudoaneurysm was appeared to be associated with significant in-stent restenosis in stented area of our patient.

The patient in the present case refused to receive any further treatment initially. We are of opinion that elderly inoperable patients or those who refuse any further interventions for coronary aneurysm, like in our case, should be managed medically to prevent thromboembolic complications. Earlier reports in this milieu also recommend the use of antiplatelet therapy (with aspirin or clopidogrel) or anticoagulation therapy (with warfarin) in such patients [10]-[13]. The other peculiarity of our case is the presence of a documentation of angiographic progression of the pseudoaneurysm (Figure 2 and Figure 3). To the best of our knowledge, such reports of giant pseudoaneurysm in the LCX after sirolimus-stent implantation are very rare. Our case also highlights the utility of embolization coils as minimally invasive, rapid, efficient, and safe method for the treatment of giant coronary artery pseudoaneurysms.

\section{Conclusion}

Formation of coronary artery aneurysm is rare, but significant safety concern, associated with DES implantation. In the present case, we depict angiographic documentation of pseudoaneurysm progression in the left circumflex artery, following sirolimus-eluting stent implantation, in a 47-year-old man. Despite several theories, the exact mechanism responsible for coronary pseudoaneurysm formation in our patient remains unknown. The patient was managed successfully with embolization coils. We are of opinion that embolization coils should be considered as a minimally invasive, rapid, efficient, and safe method for the treatment of giant coronary artery pseudoaneurysms.

\section{References}

[1] Kapoor, A., Batra, A., Kumar, S., Pandey, S., Agarwal, S.K. and Sinha, N. (2011) Coronary Pseudoaneurysm in a Non-Polymer Drug-Eluting Stent: A Rare Entity. Asian Cardiovascular and Thoracic Annals, 19, 407-410. http://dx.doi.org/10.1177/0218492311419766

[2] Roy, P., Ajani, A.E. and Warren, R. (2005) Percutaneous Treatment of a Giant Coronary Aneurysm Using a Symbiot Stent. Cardiovascular Revascularization Medicine, 6, 73-74. http://dx.doi.org/10.1016/j.carrev.2005.05.005

[3] Chadha, D.S., Hasija, P.K., Singh, N., Ghosh, A.K. and Malani, S.K. (2014) Endovascular Management of Giant Coronary Artery Aneurysm. Medical Journal Armed Forces India, 71, S242-S244. 
[4] Imai, M., Kadota, K., Goto, T., Fujii, S., Yamamoto, H., Fuku, Y. and Mitsudo, K. (2011) Incidence, Risk Factors, and Clinical Sequelae of Angiographic Peri-Stent Contrast Staining after Sirolimus-Eluting Stent Implantation. Circulation, 123, 2382-2391. http://dx.doi.org/10.1161/CIRCULATIONAHA.110.003459

[5] Hajlaoui, N. (2011) PP-024: Coronary Artery Aneurysm after Drug Eluting Stent Implantation: Case Report and Review of the Literature. International Journal of Cardiology, 147, S110. http://dx.doi.org/10.1016/s0167-5273(11)70308-4

[6] Caruso, M., Evola, S., Fattouch, K., Bracale, U.M., Incalcaterra, E., La Franca, E., et al. (2011) Chest Pain Due to Late Huge Coronary Pseudoaneurysm Following Stent Implantation. Internal Medicine, 50, 577-579.

[7] Vaghetti, M. and Palmieri, C. (2013) A Coronary Pseudoaneurysm within a Restenotic Stent Treated by Implantation of a Pericardium-Covered Stent and Drug-Eluting Balloon. Journal of Invasive Cardiology, 25, E93-E95.

[8] Terasawa, A., Yokoi, T. and Kondo, K. (2013) Stent-Assisted Coil Embolization of Coronary Artery Aneurysm. Journal of Invasive Cardiology, 25, E175-E177.

[9] Win, H.K., Polsani, V., Chang, S.M. and Kleiman, N.S. (2012) Stent-Assisted Coil Embolization of a Large Fusiform Aneurysm of Proximal Anterior Descending Artery: Novel Treatment for Coronary Aneurysms. Circulation: Cardiovascular Interventions, 5, e3-e5. http://dx.doi.org/10.1161/CIRCINTERVENTIONS.111.966754

[10] Siegrist, P.T., Sumitsuji, S., Osuga, K., Sakaguchi, T., Tachibana, K. and Nanto, S. (2013) Endovascular Coil Embolization of Behcet Disease-Related Giant Aneurysm of the Right Coronary Artery after Failure of Surgical Suture. JACC: Cardiovascular Interventions, 6, e31-e32. http://dx.doi.org/10.1016/j.jcin.2012.12.134

[11] Crawley, P.D., Mahlow, W.J., Huntsinger, D.R., Afiniwala, S. and Wortham, D.C. (2014) Giant Coronary Artery Aneurysms: Review and Update. Texas Heart Institute Journal, 41, 603-608. http://dx.doi.org/10.14503/THIJ-13-3896

[12] Boyer, N., Gupta, R., Schevchuck, A., Hindnavis, V., Maliske, S., Sheldon, M., et al. (2014) Coronary Artery Aneurysms in Acute Coronary Syndrome: Case Series, Review, and Proposed Management Strategy. Journal of Invasive Cardiology, 26, 283-290.

[13] Lima, B., Varma, S.K. and Lowe, J.E. (2006) Nonsurgical Management of Left Main Coronary Artery Aneurysms: Report of 2 Cases and Review of the Literature. Texas Heart Institute Journal, 33, 376-379. 